\title{
Contributions to Multidisciplinary Team Meetings in Cancer Care: Predictors of Complete Case Information and Comprehensive Case Discussions
}

\author{
Jessica Wihl (D) ${ }^{1-3}$ \\ Linn Rosell $\mathbb{D}^{1,2}$ \\ Kirsten Frederiksen (iD) ${ }^{4}$ \\ Sara Kinhult ${ }^{1,3}$ \\ Gert Lindell ${ }^{5}$ \\ Mef Nilbert $\mathbb{I D}^{1,4,6}$ \\ 'Department of Clinical Sciences Lund, \\ Division of Oncology and Pathology, \\ Lund University, Lund, Sweden; ${ }^{2}$ Regional \\ Cancer Centre South, Region Skåne, \\ Lund, Sweden; ${ }^{3}$ Department of \\ Hematology, Oncology and Radiation \\ Physics, Skåne University Hospital, Lund, \\ Sweden; ${ }^{4}$ Danish Cancer Society \\ Research Centre, Copenhagen, \\ Denmark; ${ }^{5}$ Department of Surgery, Skåne \\ University Hospital, Lund, Sweden; \\ ${ }^{6}$ Clinical Research Centre, Hvidovre \\ University Hospital and Copenhagen \\ University, Copenhagen, Denmark
}

Correspondence: Jessica Wihl

Department of Clinical Sciences Lund, Division of Oncology and Pathology, Lund University, Scheelev. 2, Lund, 223 63, Sweden

Email jessica.wihl@med.lu.se
Purpose: Multidisciplinary team (MDT) meetings integrate complex information and base recommendations for clinical management on interdisciplinary and multiprofessional decision-making. To support high-quality decision-making and define key performance indicators, we aimed to determine completeness of case information and contributions to MDT case discussions in cancer care.

Methods: In a prospective observational study design, based on three MDTs, we applied the Metric for Observation of Decision-Making (MODe) tool to assess the quality of case presentation and team members' contributions to case discussions. The MDTs handled patients with brain tumors, soft tissue sarcomas and hepatobiliary cancers. The results were correlated to patient and team characteristics and to MDT leadership skills.

Results: Data were collected from 349 case discussions during 32 MDT meetings. Information on radiology received the highest scores, followed by case history and information on histopathology. Patient-related information was less frequently mentioned and generally received low scores. Contributions to the case discussions were predominantly by the chair, surgeons, and oncologists with limited contributions from nurses. Leadership skills showed a positive correlation with case presentations scores and failure to reach a treatment recommendation correlated with lower case discussion scores.

Conclusion: Considerable resources are spent on MDT meetings in cancer care, which motivate initiatives to ensure high-quality and efficient decision-making processes. We identify unbalanced contributions from team members during MDT meetings, demonstrate limited provision of patient-related information and show that leadership skills may positively influence the quality of the case presentations. We suggest that MDTs should consider and develop these aspects to ensure high-quality MDT-based case management and decisionmaking.

Keywords: tumor board, cancer conference, decision-making, patient-centred, comorbidity

\section{Plain Language Summary}

Multidisciplinary team (MDT) meetings gather experts from various fields to provide the best possible recommendation for diagnosis and treatment of cancer. We mapped team members' contributions to case information and case discussions for patients with brain tumors, soft tissue sarcomas and hepatobiliary cancers. We identified unbalanced contributions from team members with a focus on medical perspectives and limited patient-related information and show that strong leadership skills may positively influence the quality of the case presentations. 


\section{Introduction}

In cancer care, formulation of the best possible treatment recommendation calls for integration of complex information, contributions from various professions, input based on updated knowledge and clinical guidelines and attention to patient perspectives. To meet these needs multidisciplinary team (MDT) meetings that match team members with tasks and responsibilities to exchange expertise have been broadly implemented. Core members include surgeon, oncologist, radiologist, pathologist, nursing staff and MDT coordinator. High-quality case management that supports effective MDT decision-making requires access to relevant information, structured case presentations, leadership skills and a meeting infrastructure that supports and encourages balanced contribution from team members. ${ }^{1-3}$ Evidence for clinical benefit is limited, though patients managed at MDT meetings have been demonstrated to be more appropriately staged and be more likely to receive treatment according to clinical guidelines. ${ }^{4,5}$ A possible influence on patient outcome remains uncertain and may vary between diagnoses with positive effects documented in eg, colorectal cancer, esophageal cancer, breast cancer, lung cancer and head and neck cancer. ${ }^{3-8}$ Several studies suggest that complex cases may be especially relevant for MDT-based decision-making with altered recommendations for clinical management in up to half of these cases, though also individual patient risk profiles and MDT performance influence patient benefit. $^{1,4,8-10}$

To support assessment of MDT performance and function, several observational assessment instruments have been developed. ${ }^{11}$ The MDT metric for the observation of decision-making (MDT-MODe) instrument assesses informationsharing and team members' contributions to case discussions and provision of treatment recommendations. ${ }^{12-14}$ MTDMODe has been applied in several countries and in various diagnostic settings, including breast cancer, lung cancer, gastrointestinal cancer, urological cancer and rare tumors to document elements of decision-making and define factors of importance for treatment recommendations. ${ }^{11,14-22}$ Chairing skills is one of the parameters evaluated in MODe, but the A Tumor Leadership Assessment (ATLAS) instrument has been developed to provide a more granular picture of MDT leadership aspects. ${ }^{23}$ Though an increasing number of factors that affect team processes have been identified, heterogenous study designs, different data collection formats and variable clinical settings hamper definition of key performance indicators. ${ }^{11}$
With the objective to map team performance in cancerrelated MDT meetings, investigate contributions to case presentations and team members participation in case discussions in new contexts, ie, other diagnostic areas and another health care setting, we applied the MDT-MODe instrument to MDT meetings for brain tumors, sarcoma and hepatobiliary cancer in Swedish health care. Further, we aimed to define predictors of efficient MDT discussions and therefore correlated the observations to patientrelated factors and scores from the ATLAS instrument.

\section{Materials and Methods}

\section{Study Design}

The study was designed as a prospective observational study.

\section{Study Paticipants}

Four MDT teams in cancer care were invited to the study through contact to the team chair who further investigated team members' interest for and practical possibilities for study participation. These teams were selected based on complete MDTs with various professions and disciplines participating, solid and long-term MDT structures, a similar number of weekly case discussions and an interest for MDT meeting evaluation and development. All teams responded positively and the study group selected the three MDTs for brain tumors, soft tissue sarcoma and hepatobiliary cancer to cover various diagnostic areas. The teams had independently developed referral guidelines that specify required clinical information and current therapeutic considerations. Two of the teams included responsible physicians and nurses in regional hospitals by video-based participation. All three selected MDTs had a high degree of complex cases with challenges related to differential diagnostics, and advanced and alternative treatment options including surgery, radiotherapy, medical oncology, and palliative care. None of the teams had applied formal frameworks such as checklists, instruments, or formal evaluation to assess MDT performance.

\section{Study Setting}

The study was set in Swedish cancer care. The MDTs that participated in the study had been running for minimum 10 years, held weekly meetings and were complete teams as regards participation from various professions and disciplines. The hospital setting was a university hospital with specialized health care responsibilities for a population of 
1.9 million. Swedish standards of care define required MDT participants that include surgeon, oncologist, pathologist, radiologist and specialized nurses, but does not provide detailed guidelines for which information should be available and does not define meeting participants' roles and responsibilities.

\section{Data Collection}

The development process for the MDT-MODe instrument documented construct validity, content validity and face validity. $^{12-14}$ The instrument collects data in two sections based on 6 parameters (case history, radiology, pathology, psychosocial issues, comorbidities and patient's views and preferences) that define information during the case presentations and 8 parameters (input from the chair, surgeon, physician, oncologist, radiologist, pathologist, nurse and MDT coordinator) that define team members' contributions to the case discussions. Each variable is scored from 1 to 5 on a Likert-type rating scale. We calculated the composite score independently for each section, ie, case information scores that can range from 6 to 30 and case discussion scores that can range from 8 to 40 . Further, the tool collects information on whether a treatment recommendation was provided, classified as yes/no.

During a 6-month period, we observed 32 MDT meeting with 349 case discussions for patients with brain tumors, soft tissue sarcomas and hepatobiliary cancers and collected data on case presentations and case discussions using the MDTMODe tool. Team characteristics are presented in Table 1 and all teams were complete with participation from eg, surgeons, orthopedic surgeons (for sarcoma), neurosurgeons (for brain tumors), oncologists, radiologists, pathologists, and nurses. The teams did, however, not include MDT coordinators. Data were collected based on a non-participant observational approach where the three observers (two oncologists and one oncology nurse) who were not part of the MDT team and had previous experience from observational MDT assessment. Registrations by two independent observers were made for 104 of 349 cases at 7 of the 32 MDTs (38 case discussions on brain tumors, 30 on soft tissue sarcoma and 36 on hepatobiliary cancer). Investigations of interobserver reliability weighted double observations by 0.5 and did not give priority to any of the observers.

Clinical data including age and sex were collected during the case presentations. Measures were taken to reduce bias; the observers did not participate in the meeting and to reduce interference were physically observing from the back of the room and to document inter-observer agreement observations were performed by two independent observers in onethird of the cases discussions. Further, all observers had previously used the instrument and were familiar with the scoring system, which followed the predefined descriptions for the different scores. Data on leadership skills based on the ATLAS instrument were obtained from data collected in an earlier study in the same MDT meetings. ${ }^{24}$

\section{Statistical Analyses}

MDT characteristics are presented using descriptive statistics. Data processing and statistical analysis used the SAS statistical software release 9.4. Inter-observer reliability was assessed using the parametric intraclass correlation coefficient (ICC) and the weighted Kappa statistics; and inter-observer agreement was assessed by mean differences with 95\% limits of agreement and proportion with differences of more than two scale points. The distribution of scores according to patient and team characteristics (eg, sex, age, case order, leadership scores) was assessed using linear mixed models with observer, patient and MDT as random components. To correlate MDT-MODe data with case order during meetings, data were dichotomized from cases 1-9 vs later and for correlations to leadership skills ATLAS scores were dichotomized at the median.

\section{Results \\ Data Set}

Data were collected from 349 case presentations and case discussions (110 brain tumors, 117 soft tissue sarcomas and 122 hepatobiliary cancers) during 32 MDT meetings. An

Table I Summary Data on MDT Teams Studied

\begin{tabular}{|c|c|c|c|c|c|}
\hline MDT Team & $\begin{array}{c}\text { Number of Case } \\
\text { Discussions } \\
\text { Observed }\end{array}$ & $\begin{array}{c}\text { Number of MDT } \\
\text { Meetings } \\
\text { Observed }\end{array}$ & $\begin{array}{c}\text { Mean (Range) } \\
\text { Number of } \\
\text { Participants }\end{array}$ & $\begin{array}{c}\text { Mean (Range) } \\
\text { Number of Case } \\
\text { Discussions }\end{array}$ & $\begin{array}{l}\text { Mean Time per } \\
\text { Case Discussion }\end{array}$ \\
\hline Brain tumors & 110 & II & $12(7-16)$ & $10(6-17)$ & $5.7 \mathrm{~min}$ \\
\hline Soft tissue sarcoma & 117 & II & $10(6-14)$ & II (4-I8) & $5.0 \mathrm{~min}$ \\
\hline Hepatobiliary cancer & 122 & 10 & $10(7-13)$ & $12(7-16)$ & $7.9 \mathrm{~min}$ \\
\hline
\end{tabular}






Figure I Box plot showing MDT-MODe scores for the 14 parameters evaluated. From left to right and mean/median scores: case presentation parameters case history (3.4/ $3)$, radiology (4.7/5) and pathology (2.5/3) and patient-related factors that include psychologic status (I.4/I), comorbidity (I.9/I) and patient's view (I.I/I) and case discussion with participation from chair (3.7/4), surgeon (3.6/4), physician (3.I/I), oncologist (3.I/3), nurse (I.3/I), radiologist (3.4/4), pathologist (I.5/I) and MDT coordinator (I.I/I).

overview of the distribution of scores based on the MDTMODe instrument is provided in Figure 1 and in Supplementary Figure 1. Since the MDTs observed did not contain MDT coordinators and the evaluations of physicans were not consistent, data from these categories were not used in the further correlation analyses. Patient characteristics were $155(44 \%)$ female, 129 (37\%) 70 years or older and $82(23 \%)$ cases listed and discussed beyond case number 10 during the meeting. Overall, treatment recommendations were provided in $311(92 \%)$ of the case discussions.

\section{MDT-MODe Scores}

Mean scores above 3 were for case presentations achieved for case history and radiology and were for case discussion achieved for contributions from the chair, surgeons, physicians, oncologists and radiologists (Figure 1, Supplementary Figure 1). Low scores applied to information on patient-related factors (psychosocial factors, comorbidity, and patients' views) and to contributions from nurses. High (4-5) scores were obtained for radiologic information in $92 \%$ of the case presentations, for case histories in $44 \%$ and for pathological information in $18 \%$. Information on patient-related aspects received low scores (1-2) in 72-95\% of the case presentations. Contributions to the case discussions were scored as high (4-5) for chairs in $62 \%$ of the discussions, for surgeons in $61 \%$ for radiologists in $60 \%$ and for oncologists in $50 \%$ of the discussions. High case discussion scores for nurses' participation were, however, only found in $4 \%$ of the cases.

\section{Inter-Rater Reliability}

Double observations were available in 104 case discussions. ICCs ranged from 0.00 to 0.80 and kappa values from 0.00 to 0.64 and absolute mean differences from 0.03 to 0.94 and 
percentage with score difference of more than 2 in $0 \%$ to 13.5\% (Supplementary Table 1).

\section{Correlation to Patient and Team Characteristics}

The mean and the total composite scores differed between the teams with mean scores for case presentations of 16.5 in brain tumors, 14.5 in hepatobiliary cancer and 14.0 in soft tissue sarcoma and corresponding composite scores for case discussions of 17.7, 17.3 and 15.7, respectively. Since our primary aim was not to study differences between the teams, further analyses were performed based on the entire data set. The results from analyses in relation to patient and team characteristics are available in Table 2 . Whereas patient sex and age did not significantly influence the results, cases discussed later during the conference (ie, listed from case 10 and later) showed a tendency for lower contributions to the case presentations (mean difference $-0.7, p=0.06$ ). Case discussions that did not reach a treatment recommendation showed significantly lower scores (mean difference -1.2 , $\mathrm{p}=0.04$ ). When the results from MDT-MODe were correlated to data on leadership skills, ATLAS scores above the median correlated with higher case presentation scores (mean difference $0.84, \mathrm{p}<0.001$ ) and showed borderline effect for case discussion scores (mean difference $0.61, \mathrm{p}=0.06$ ).

\section{Discussion}

With the aims to map team performance in cancer-related MDT meetings and to investigate contributions to case presentations and team members participation in case discussions in new contexts, we applied the MDT-MODe instrument to MDT meetings for brain tumors, soft tissue sarcoma and hepatobiliary cancer in Swedish cancer care and investigated correlations to patient-related factors and leadership scores from the ATLAS instrument. Case presentations during MDT meetings provide an opportunity to review and re-evaluate imaging, pathology and patientrelated factors based on access to relevant data and active participation from team members to achieve high-quality decision-making. ${ }^{3}$ The increasing demand for MDT case discussions has stimulated research on team-based decision-making, team performance and implementation of MDT recommendations and has led to development of various observational tools to support data collection. ${ }^{11,25,26}$

The MDT-MODe instrument provides an evidencebased and objective measure that has been applied to define comprehensiveness of patient profiles and team members' contributions to case discussions in several diagnostic areas (Supplementary Table 2). ${ }^{12-14}$ Our observed mean scores for contributions to case presentations fit well with observations in MDTs for colorectal cancer and breast cancer with mean scores of 3.9-4.6 for patient history, 3.6-4.2 for radiology and 3.8-4.4 for pathology. ${ }^{14,18,20}$ Also, the contributions to the case discussions with mean scores of 3.2-3.7 for chair, surgeon, oncologist, and radiologist with lower mean scores of 1.41.6 for pathologists and nurses are in line with observations from MDTs in colorectal cancer, breast cancer and ovarian cancer with mean scores of $4.7-4.8$ for surgeons'

Table 2 Mean Composite Scores for Case Presentation and Case Discussion in Relation to Patient Characteristics

\begin{tabular}{|c|c|c|c|c|c|}
\hline & $\begin{array}{l}\text { Individuals/ } \\
\text { Observations }\end{array}$ & $\begin{array}{c}\text { Mean (Range) Score Case } \\
\text { Presentation }\end{array}$ & p-value & $\begin{array}{c}\text { Mean (Range) Score Case } \\
\text { Discussion* }\end{array}$ & p-value \\
\hline Overall mean & $349 / 453$ & $14.9(8-23)$ & & $16.9(6-26)$ & \\
\hline Female & I55/207 & I4.8 (8-23) & & $16.7(8-25)$ & \\
\hline Male & $194 / 246$ & $15.0(9-22)$ & 0.39 & $17.0(6-26)$ & 0.71 \\
\hline Patient age $\leq 70$ years & $211 / 280$ & $15.0(9-23)$ & & I7.1 (6-26) & \\
\hline Patient age $>70$ years & $129 / 162$ & I5.| (8-2|) & 0.11 & $16.8(8-23)$ & 0.52 \\
\hline Early discussion (cases I-I0) & $267 / 337$ & I5.| (9-23) & & $16.9(6-26)$ & \\
\hline $\begin{array}{l}\text { Late discussion (cases II and } \\
\text { beyond) }\end{array}$ & $82 / 116$ & 14.4 (8-19) & 0.06 & $16.7(9-23)$ & 0.17 \\
\hline $\begin{array}{l}\text { Treatment recommendation } \\
\text { provided }\end{array}$ & $311 / 405$ & $15.0(8-23)$ & & $16.9(7-26)$ & \\
\hline $\begin{array}{l}\text { Treatment recommendation } \\
\text { not provided }\end{array}$ & $26 / 35$ & $13.9(9-22)$ & 0.08 & $15.7(6-23)$ & 0.04 \\
\hline ATLAS score (high) & $208 / 274$ & $15.2(8-23)$ & & $17.0(6-23)$ & \\
\hline ATLAS score (low) & $14|/| 79$ & $14.5(9-22)$ & $<0.001$ & $16.6(7-26)$ & 0.06 \\
\hline
\end{tabular}

Note: *Excluding data from physician and MDT coordinator. 
contributions, 3.2-4.4 for radiologists, 4.5 for pathologists, 3.1 for oncologists and 4.1 for chairs. ${ }^{19-21}$ Hence, case history, radiologic information and contributions from chairs, surgeons, radiologists, and oncologists seem to be key drivers of MDTs' decision-making. ${ }^{27}$

Low scores for information on psychosocial factors, comorbidities and consideration of patient views and limited roles for nursing staff have also been documented by other investigators with mean scores of 1.7-2.8 for psychological factors, 1.4-3.4 for comorbidity and 1.5-2.5 for patients' views. ${ }^{19-22}$ Missing information on patientrelated aspects is problematic considering the complex treatment options with considerable risks and side-effects that are discussed during the MDT meetings and suggested to the patient. Our observations add to the increasing literature on limited attention to patient-related factors during MDT meetings. ${ }^{19-22,28,29}$ We did, however, not find any differences in whether psychosocial factors and patient preferences were considered in relation to patient age, which contrasts with reports on limited patient-centeredness in older patients. ${ }^{30}$ Our observations suggest that nurses' roles in MDT meetings could be more explicit. Active involvement of care professionals in MDT discussions could voice psychologic and patient-related aspects and thereby support patient-centered decision-making, which may in turn increase the likelihood of acceptance of the recommendations provided. ${ }^{17,31,32}$

In the MDT meetings studied, treatment recommendations were provided in $92 \%$ of the case discussions. This compares well with other reports on provision of treatment recommendations in $74-94 \%$ of the cases. ${ }^{14,16-18}$ Failure to reach a treatment recommendation was associated with significantly lower scores for case discussions and showed a trend for an effect also on case presentation scores (Table 2). Correlations between MDT-MODe scores and decision-making have been reported from other diagnostic areas and have suggested that quality of case histories and radiology information are the major determinants of the team reaching a treatment recommendation. ${ }^{13,16}$

Leadership skills are essential to mobilize teams to effective meeting structures. Encouraging involvement from team members with diverse competences and personalities may, however, be challenging in large, multiprofessional teams that also experience increasing case loads and resource constraints. ${ }^{33}$ Based on data from a recent study on leadership and chairing skills, we identified a significant correlation between leadership scores and case presentation scores, and we observed a trend for correlation also to the case discussion scores (Table 2). This observation merits further investigation into how leadership aspects may influence quality of case presentations and contributions to case discussions.

Increasing case load that lead to prolonged MDT meetings risk decision-making fatigue, which is supported by observations of reduced quality of information and weaker contributions to case discussions in the latter part of the MDT meetings. ${ }^{33-35}$ MDTs seek solutions to handle resource tensions eg, through mid-meeting breaks, meetings twice weekly, pre-MDT conferences with participation from a reduced number of experts to handle standard cases according to guidelines and strategies to prioritize complex cases for full MDT discussions. The MDT meetings here studied did generally last one to one and a half hours with a maximum of 18 ses. Despite relatively modest case load, we observed a tendency for lower case presentation scores beyond case 10, which serves as a reminder of attention to quality in the later parts of MDT meetings.

Strengths and limitations apply to our study. Though our findings are not new, the findings validate previously observed patterns of contributions to case presentations and participations in case discussions at MDT meetings. In this regard our findings demonstrate replicability using the MDT-MODe instrument in new health care contexts related to diagnoses and country. Currently available data, are however, largely derived from the Western world, which limits generalizability and signifies needs for studies also from other health care settings around the world. Further, MDT meeting structures, case load and participating health professionals vary between diagnoses and teams, which may limit generalizability though our composite scores fit well with the results from other teams. Though the MDTMODe instrument provides an overview of team members' input and involvement in the decision-making process, it does not provide information on team performance eg, related to communication and interaction, discussion climate or quality of decision-making. Since we excluded physicians and MDT coordinators from the composite score calculations, the exact scores are not directly comparable with other studies. Reliability of MDT-MODe has been demonstrated to be favorable, albeit with some variability between the parameters. Further, if most responses are within one category and show strong agreement, reliability measures are less informative. Further, we did not discriminate between standard or complex cases, which could have been relevant to address to investigate whether complex 
cases received increased attention with more comprehensive case presentations and case discussions. The risk of Hawthorn effect (teams changing their usual behavior when observed), cannot be excluded, though it was the observers' experience that this was not relevant, and it was deliberately reduced through the observers sitting at the back of the room and the MDT participants not being informed about the detailed aims of the study and parameters within the observational tool.

\section{Conclusion}

We identify patient history and radiology as predominant the information sources during case presentations and find that contributions from the chair, surgeon, radiologists, and oncologist are the key drivers for treatment recommendations. Unbalanced contributions to case presentations and case discussions merits increased attention to team members' contributions, not least to ensure relevant consideration of patients' perspectives. MDT leadership skills correlated with higher scores for case presentations and failure to reach a treatment recommendation correlated with lower case discussion scores. Our findings suggest that focus on these aspects may be relevant to ensure effective and robust MDT decision-making.

\section{Data Sharing Statement}

Raw data are available from the lead author (Jessica Wihl) upon request.

\section{Ethic's Approval and Informed Consent}

All patient data were anonymized and are presented at group level. The study was ethically reviewed and granted permission by the regional ethics committee at Lund University (registration numbers 2016-195 and 2019-04254) and was based on written informed consent from the team leader on behalf of the team and oral information to team members on the observational assessment. At the end of the study, all teams were provided with feedback on the results and scores.

\section{Acknowledgments}

The study was financially supported by the Swedish Cancer Society and by the Regional Cancer Center South, Region Skåne.

\section{Author Contributions}

All authors made substantial contributions to conception and design, acquisition of data, or analysis and interpretation of data; took part in drafting the article or revising it critically for important intellectual content; agreed to submit to the current journal; gave final approval of the version to be published; and agree to be accountable for all aspects of the work.

\section{Funding}

Funding was obtained from the Kamprad foundation and support for research staff was provided by the Regional Cancer Center South, Region Skåne.

\section{Disclosure}

The authors report no conflicts of interest in this work.

\section{References}

1. Lamb BW, Brown KF, Nagpal K, Vincent C, Green JSA, Sevdalis N. Quality of care management decisions by multidisciplinary cancer teams: a systematic review. Ann Surg Oncol. 2011;18(8):2116-2125. doi:10.1245/s10434-011-1675-6

2. Taylor C, Atkins L, Richardson A, Tarrant R, Ramirez AJ. Measuring the quality of MDT working: an observational approach. $B M C$ Cancer. 2012;12:202. doi:10.1186/1471-2407-12-202

3. Croke JM, El-Sayed S. Multidisciplinary management of cancer patients: chasing a shadow or real value? An overview of the literature. Curr Oncol. 2012;19(4):e232-8. doi:10.3747/co.19.944

4. Pillay B, Wootten AC, Crowe H, et al. The impact of multidisciplinary team meetings on patient assessment, management and outcomes in oncology settings: a systematic review of the literature. Cancer Treat Rev. 2016;42:56-72. doi:10.1016/j.ctrv.2015.11.007

5. El Saghir NS, Keating NL, Carlson RW, Khoury KE, Fallowfield L. Tumor boards: optimizing the structure and improving efficiency of multidisciplinary management of patients with cancer worldwide. American Society of Clinical Oncology educational book. American Society of Clinical Oncology. Annual Meeting; 2014:e461-6.

6. Heinke MY, Vinod SK. A review on the impact of lung cancer multidisciplinary care on patient outcomes. Transl Lung Cancer Res. 2020;9(4):1639-1653. doi:10.21037/tlcr.2019.11.03

7. Basta YL, Bolle S, Fockens P, Tytgat K. The value of multidisciplinary team meetings for patients with gastrointestinal malignancies: a systematic review. Ann Surg Oncol. 2017;24(9):2669-2678. doi:10.1245/s10434-017-5833-3

8. Prades J, Remue E, van Hoof E, Borras JM. Is it worth reorganising cancer services on the basis of multidisciplinary teams (MDTs)? A systematic review of the objectives and organisation of MDTs and their impact on patient outcomes. Health Policy (New York). 2015;119(4):464-474. doi:10.1016/j.healthpol.2014.09.006

9. Back MF, Ang EL, Ng WH, et al. Improvements in quality of care resulting from a formal multidisciplinary tumour clinic in the management of high-grade glioma. Annals Acad Med Singapore. 2007;36 (5):347-351.

10. Rogers MJ, Matheson L, Garrard B, et al. Comparison of outcomes for cancer patients discussed and not discussed at a multidisciplinary meeting. Public Health. 2017;149:74-80. doi:10.1016/j.puhe.2017. 04.022 
11. Soukup T, Lamb BW, Arora S, Darzi A, Sevdalis N, Green JS. Successful strategies in implementing a multidisciplinary team working in the care of patients with cancer: an overview and synthesis of the available literature. $J$ Multidisc Healthc. 2018;11:49-61. doi:10.2147/JMDH.S117945

12. Lamb BW, Wong HWL, Vincent C, Green JSA, Sevdalis N. Teamwork and team performance in multidisciplinary cancer teams: development and evaluation of an observational assessment tool. BMJ Qual Saf. 2011;20(10):849-856. doi:10.1136/bmjqs.2010. 048660

13. Lamb BW, Sevdalis N, Benn J, Vincent C, Green JS. Multidisciplinary cancer team meeting structure and treatment decisions: a prospective correlational study. Ann Surg Oncol. 2013;20 (3):715-722. doi:10.1245/s10434-012-2691-x

14. Jalil R, Akhter W, Lamb BW, et al. Validation of team performance assessment of multidisciplinary tumor boards. J Urol. 2014;192 (3):891-898. doi:10.1016/j.juro.2014.03.002

15. Seretis C, Mankotia R, Goonetilleke K, Rawstorne E. Quality assessment of decision-making in colorectal cancer multidisciplinary meetings. J BUON. 2014;19(4):913-916.

16. Soukup T, Lamb BW, Sarkar S, et al. Predictors of treatment decisions in multidisciplinary oncology meetings: a quantitative observational study. Ann Surg Oncol. 2016;23(13):4410-4417. doi:10.1245/ s10434-016-5347-4

17. Hahlweg P, Didi S, Kriston L, Härter M, Nestoriuc Y, Scholl I. Process quality of decision-making in multidisciplinary cancer team meetings: a structured observational study. BMC Cancer. 2017;17 (1):772. doi:10.1186/s12885-017-3768-5

18. Lumenta DB, Sendlhofer G, Pregartner G, et al. Quality of teamwork in multidisciplinary cancer team meetings: a feasibility study. PLoS One. 2019;14(2):e0212556. doi:10.1371/journal.pone.0212556

19. Gandamihardja TAK, Soukup T, McInerney S, Green JSA, Sevdalis N. Analysing breast cancer multidisciplinary patient management: a prospective observational evaluation of team clinical decision-making. World J Surg. 2019;43(2):559-566. doi:10.1007/ s00268-018-4815-3

20. Scott R, Hawarden A, Russell B, Edmondson RJ. Decision-making in gynaecological oncology multidisciplinary team meetings: a cross-sectional, observational study of ovarian cancer cases. Oncol Res Treat. 2020;43(3):70-77. doi:10.1159/000504260

21. Shah S, Arora S, Atkin G, et al. Decision-making in colorectal cancer tumor board meetings: results of a prospective observational assessment. Surg Endosc. 2014;28(10):2783-2788. doi:10.1007/ s00464-014-3545-3

22. Rosell L, Wihl J, Hagberg O, Ohlsson B, Nilbert M. Function, information, and contributions: an evaluation of national multidisciplinary team meetings for rare cancers. Rare Tumors. 2019;11:2036361319841696. doi:10.1177/2036361319841696

23. Jalil R, Soukup T, Akhter W, Sevdalis N, Green JSA. Quality of leadership in multidisciplinary cancer tumor boards: development and evaluation of a leadership assessment instrument (ATLAS). World J Urol. 2018;36(7):1031-1038. doi:10.1007/s00345-0182255-1

Journal of Multidisciplinary Healthcare

\section{Publish your work in this journal}

The Journal of Multidisciplinary Healthcare is an international, peerreviewed open-access journal that aims to represent and publish research in healthcare areas delivered by practitioners of different disciplines. This includes studies and reviews conducted by multidisciplinary teams as well as research which evaluates the results or conduct of such teams or healthcare processes in general. The journal

Submit your manuscript here: https://www.dovepress.com/journal-of-inflammation-research-journal
24. Wihl J, Rosell L, Bendahl PO, et al. Leadership perspectives in multidisciplinary team meetings; observational assessment based on the ATLAS instrument in cancer care. Cancer Treat Res Commun. 2020;25:100231. doi:10.1016/j.ctarc.2020.100231

25. Ke KM, Blazeby JM, Strong S, Carroll FE, Ness AR, Hollingworth W. Are multidisciplinary trams cost-effective? A systematic review of the literature. Cost Eff Resour Alloc. 2013;11(1):7. doi:10.1186/1478-7547-11-7

26. Lamprell K, Arnolda G, Delaney GP, Liauw W, Braithwaite J. The challenge of putting principles into practice: resource tensions and real-world constraints in multidisciplinary oncology team meetings. Asia Pac J Clin Oncol. 2019;15(4):199-207.

27. Soukup T, Petrides KV, Lamb BW, et al. The anatomy of clinical decision-making in multidisciplinary cancer meetings: a cross-sectional observational study of teams in a natural context. Medicine. 2016;95(24):e3885. doi:10.1097/MD.0000000000003885

28. Lamb BW, Sevdalis N, Arora S, Pinto A, Vincent C, Green JS. Teamwork and team decision-making at multidisciplinary cancer conferences: barriers, facilitators, and opportunities for improvement. World J Surg. 2011;35(9):1970-1976. doi:10.1007/ s00268-011-1152-1

29. Hoinville L, Taylor C, Zasada M, Warner R, Pottle E, Green J. Improving the effectiveness of cancer multidisciplinary team meetings: analysis of a national survey of MDT members' opinions about streamlining patient discussions. BMJ Open Qual. 2019;8(2): e000631. doi:10.1136/bmjoq-2019-000631

30. Bolle S, Smets EMA, Hamaker ME, Loos EF, van Weert JCM. Medical decision making for older patients during multidisciplinary oncology team meetings. J Geriatric Oncol. 2019;10(1):74-83. doi:10.1016/j.jgo.2018.07.016

31. Rajan S, Foreman J, Wallis MG, Caldas C, Britton P. Multidisciplinary decisions in breast cancer: does the patient receive what the team has recommended? $B r \quad J$ Cancer. 2013;108 (12):2442-2447. doi:10.1038/bjc.2013.267

32. Jalil R, Ahmed M, Green JS, Sevdalis N. Factors that can make an impact on decision-making and decision implementation in cancer multidisciplinary teams: an interview study of the provider perspective. Int $J$ Surg. 2013;11(5):389-394. doi:10.1016/j. ijsu.2013.02.026

33. Rankin NM, Lai M, Miller D, et al. Cancer multidisciplinary team meetings in practice: results from a multi-institutional quantitative survey and implications for policy change. Asia Pac J Clin Oncol. 2018;14(1):74-83. doi:10.1111/ajco.12765

34. Soukup T, Gandamihardja TAK, McInerney S, Green JSA, Sevdalis N. Do multidisciplinary cancer care teams suffer decision-making fatigue: an observational, longitudinal team improvement study. BMJ Open. 2019;9(5):e027303. doi:10.1136/ bmjopen-2018-027303

35. Soukup T, Lamb BW, Morbi A, et al. A multicentre cross-sectional observational study of cancer multidisciplinary teams: analysis of team decision making. Cancer Med. 2020;9(19):7083-7099. doi:10.1002/cam4.3366 covers a very wide range of areas and welcomes submissions from practitioners at all levels, from all over the world. The manuscript management system is completely online and includes a very quick and fair peer-review system. Visit http://www.dovepress.com/testimonials. php to read real quotes from published authors. 